M.R. Turner, $\mathrm{PhD}$

S.R. Irani, DPhil

M.I. Leite, MD

K. Nithi, PhD

A. Vincent, FRCPath

O. Ansorge, MD

Address correspondence and reprint requests to Dr. Olaf Ansorge, Department of Neuropathology, West Wing Level 1, John Radcliffe Hospital, Oxford OX3 9DU, UK olaf.ansorge@clneuro.ox.ac.uk

\section{Editorial, page 414}

Supplemental data at www.neurology.org

\title{
Progressive encephalomyelitis with rigidity and myoclonus
}

\author{
Glycine and NMDA receptor antibodies
}

\section{ABSTRACT}

Background: The syndrome of progressive encephalopathy with limb rigidity has been historically termed progressive encephalomyelitis with rigidity and myoclonus (PERM) or stiff-person syndrome plus.

Methods: The case is presented of a previously healthy 28-year-old man with a rapidly fatal form of PERM developing over 2 months.

Results: Serum antibodies to both NMDA receptors (NMDAR) and glycine receptors (GlyR) were detected postmortem, and examination of the brain confirmed an autoimmune encephalomyelitis, with particular involvement of hippocampal pyramidal and cerebellar Purkinje cells and relative sparing of the neocortex. No evidence for an underlying systemic neoplasm was found.

Conclusion: This case displayed not only the clinical features of PERM, previously associated with GlyR antibodies, but also some of the features associated with NMDAR antibodies. This unusual combination of antibodies may be responsible for the particularly progressive course and sudden death. Neurology ${ }^{\circledR} 2011 ; 77: 439-443$

\section{GLOSSARY}

GAD = glutamic acid decarboxylase; GlyR = glycine receptor; NMDAR = NMDA receptor; PERM = progressive encephalomyelitis with rigidity and myoclonus; SPS-Plus = stiff-person syndrome plus.

A previously healthy 28-year-old man was referred to the neurology clinic after 2 suspected generalized seizures. He reported erectile failure over the preceding month. There was no history of recreational drug use and no significant family history of neurologic disease. Initial physical examination was normal. An EEG demonstrated frequent sharp waves in the left anterior temporal lobe, and he was commenced on valproate sodium $300 \mathrm{mg}$ twice daily. MRI of the brain was normal.

Three weeks later, he presented to the emergency department with increasingly stiff legs, urinary retention, and constipation. On examination, he appeared disoriented. He was afebrile. Jerky eye pursuit movements, dysarthria, and finger-nose ataxia were observed, with marked rigidity of all limbs and occasional myoclonic limb jerks with hyperekplexia. There was generalized hyperreflexia with extensor Babinski responses. Sensation was intact. A repeat EEG revealed ongoing sharp waves in the left anterior temporal lobe, with no clear correlation to the limb myoclonus.

The following investigations were normal: MRI of the whole spine, routine blood analysis including vitamin B12, thyroid function, serum ammonia and creatine kinase, serum ceruloplasmin, and copper levels. Serology for HIV 1 and 2 was negative.

Levetiracetam was commenced, but over the next 3 days his condition deteriorated rapidly, leading to intensive care admission with suspected subclinical seizures (for which IV phenytoin was administered), metabolic acidosis, ventilatory failure, hypotension, renal failure, and ultimately disseminated intravascular coagulation resulting in death.

From the Oxford University Nuffield Department of Clinical Neurosciences (M.R.T., S.R.I., M.I.L., A.V., O.A.) and Department of Neuropathology (O.A.), John Radcliffe Hospital, Oxford; and Northampton General Hospital (K.N.), Northampton, UK.

Disclosure: Author disclosures are provided at the end of the article. 

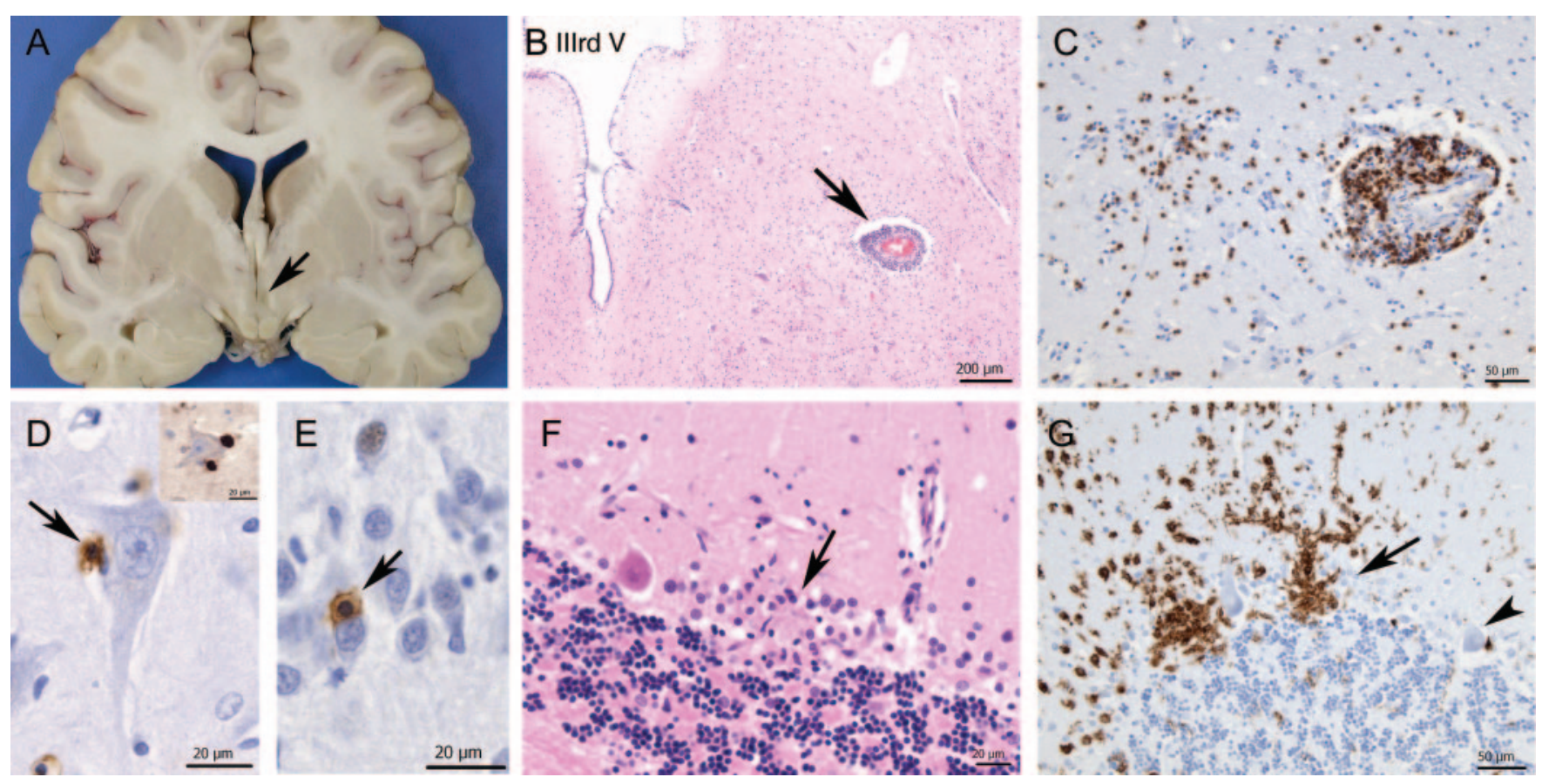

(A) Coronal section at the level of the anterior hippocampus shows a slit-like third ventricle (arrow) reflecting swelling due to edema and inflammation of the hypothalamic region (B). Most of the lymphocytic inflammation was perivascular (B, arrow). However, T lymphocytes also extended into the parenchyma (C, CD3 stain). Direct contact between T cells and neurons can be observed at high-power magnification ( $D, E$, arrows). T cells attached to a pyramidal (D) and granule neuron (E) of the hippocampus (CD3 stain) are shown. These cells were mostly of CD8 subtype (D, inset). Selective attack of individual Purkinje cells by $T$ cells/microglia was also seen in the cerebellum $(F, G)$. A microglial nodule replacing a Purkinje cell was noted $(F$, arrow) and the striking outline of 2 Purkinje cells, including proximal dendrites, by activated microglia (CD68 stain) (G, arrow). There was complete sparing of another adjacent Purkinje cell (G, arrowhead).

METHODS The brain and upper cervical cord were available for neuropathologic examination. Methodologic details are detailed elsewhere (see e-Methods on the Neurology ${ }^{\circledR}$ Web site at www.neurology.org).

RESULTS Macroscopically, there was brain swelling, particularly involving the mesial temporal lobes and hypothalamus (figure 1). Histology revealed encephalomyelitis. The inflammatory exudate was mostly perivascular and consisted of both $B$ cells $(\mathrm{CD} 20+)$ and T cells (CD3+). Quantification of perivascular lymphocytoid infiltrates in the paraventricular white matter of the temporal horn indicated a ratio of $3: 1$ of $\mathrm{T}$ cells $(\mathrm{CD} 3+)$ vs B cells $(\mathrm{CD} 20+)$. Approximately $40 \%$ of all perivascular mononuclear lymphoid cells in this location were CD8+ cytotoxic T cells. A few scattered VS38+ plasma cells were present. These were confined to the meninges and rare perivascular parenchymal spaces (figure e-1). There were no $\mathrm{CD} 20+$ or VS38 + B cells in direct apposition to neurons or glia. However, antihuman IgG staining showed patchy positivity of these cell types (figure e-1), but no significant perivascular IgG leakage. Serial sections of microglial nodules in the CA4 region of the hippocampus and Purkinje cell layer of the cerebellum revealed a ratio of $\mathrm{CD} 8+$ vs $\mathrm{CD} 4+\mathrm{T}$ cells of 3:1. CD20 $+\mathrm{B}$ cells were not a significant component of these foci (one cell seen in one microglial nodule in the cerebellum). There was no vasculitis. Gray matter was more involved than white matter and there was no demyelination. The hippocampal, hypothalamic, brainstem, and cerebellar gray matter was most severely affected, with relative sparing of the neocortex. In these areas $\mathrm{CD} 3+\mathrm{T}$ cells infiltrated the parenchyma and were found in close apposition to neurons or forming clusters, together with microglia at various stages of activation (figure 1). Further typing and examination at high magnification suggested direct attack of hippocampal pyramidal cells and cerebellar Purkinje cells by CD $8+T$ cells and CD68+ microglial cells (figure 1) with microglial nodule formation and neuronal loss. CD68+ cells with macrophage morphology were seen at the center of occasional microglial nodules and around a few parenchymal vessels. There was no hippocampal sclerosis. A screen for viral inclusions and antigens was negative. In the absence of other 
Figure 2 Glycine receptor (GlyR) and NMDA receptor (NMDAR) antibodies
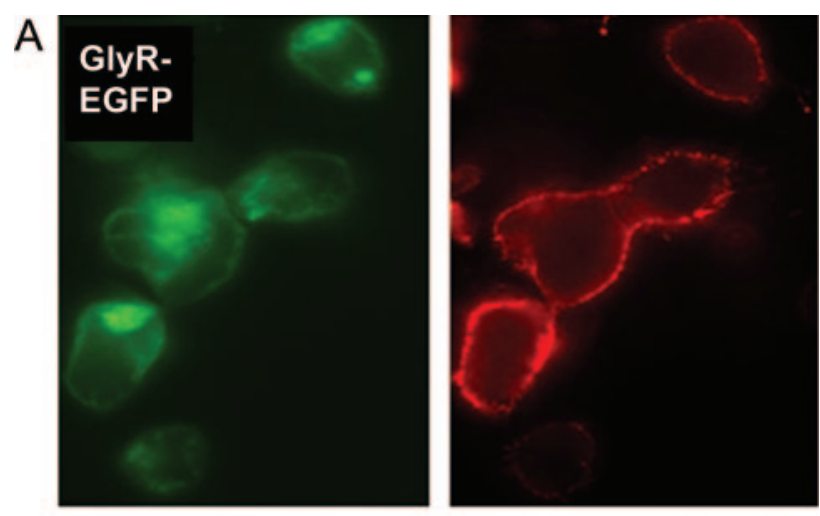

B
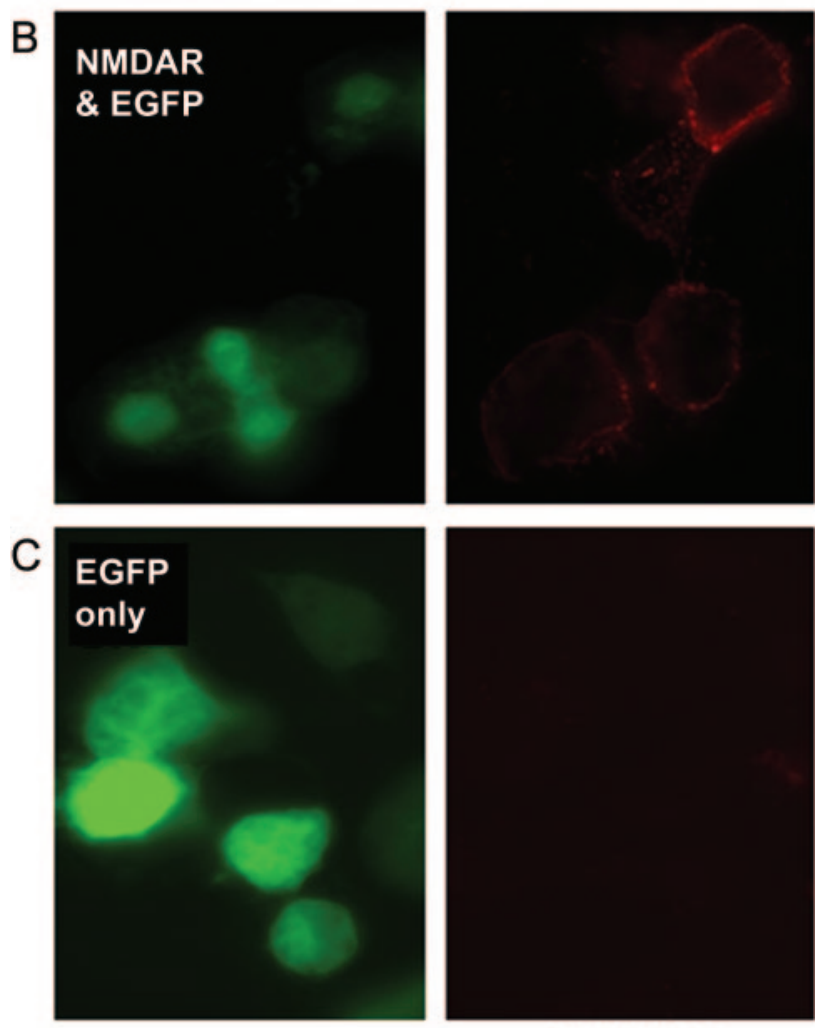

Patient IgG

HEK cells transfected with EGFP-tagged GlyR cDNA (GlyR-EGFP; A) or cotransfected with NMDARs (NR1 and NR2B subunits) plus EGFP (NMDAR and EGFP; B). EGFP fluorescence shown in green. Patient immunoglobulin $\mathrm{G}(\mathrm{lgG})$ binding shown in red: score of 3 for GlyR antibody and 2 for NMDAR antibody (scoring system as in Irani et al. ${ }^{\text {) }}$. Patient IgG did not bind to HEK cells transfected with EGFP only (C).

diagnostic findings, the picture was suggestive of an autoimmune encephalomyelitis. A systemic postmortem examination did not identify a neoplasm or other specific pathology.

Retrospective analysis of premortem serum revealed antibodies to both the glycine receptor (GlyR; score 3 on scale $0-4$, normal range $<1^{1}$ ) and NMDA receptor (NMDAR; score 2 on scale $0-4$, normal range $<1^{2}$ ). Serum did not bind to untransfected HEK or other antigen-expressing cells (figure 2). Antineuronal (Hu, Yo, Ri, CV2, amphiphysin), glutamic acid decarboxylase (GAD), and voltage-gated potassium channel complex antibodies were negative on routine screening.

DISCUSSION This young man had a rapidly progressive, multifocal, and fatal syndrome characterized by encephalopathy, myoclonus, cerebellar ataxia, and marked limb rigidity. Since the earliest descriptions of this disorder, ${ }^{3}$ it has been variably termed progressive encephalomyelitis with rigidity and myoclonus (PERM) ${ }^{4}$ or stiff-person syndrome plus (SPS-Plus). 5 This syndrome has been previously associated with GlyR but not NMDAR antibodies. ${ }^{1}$

A similar disorder was described in a 19-year-old subject $^{4}$ (case 2), in which the symptoms were initially interpreted as nonorganic. Sudden death is also a previously recognized outcome, and was linked in another case to metabolic acidosis with respiratory compromise. ${ }^{6}$ Frank seizures were not reported in either case, and the descriptions of the limb "spasms" reported in both cases were reminiscent of hyperekplexia (see also reference ${ }^{1}$ ). A case series characterized SPS-Plus as those cases of rigidity with superimposed encephalomyelitis. ${ }^{5}$ Additional features beyond "classic" SPS noted by these authors were generalized rigidity in the limbs as well as paraspinal muscles, long-tract signs, autonomic and sphincter involvement, and cognitive change reflected in gray matter involvement on postmortem examination. They noted both paraneoplastic (breast and smallcell lung carcinomas, with amphiphysin antibodies) and nonparaneoplastic cases, with the absence of the GAD antibodies frequently found in "uncomplicated" SPS. Within SPS-Plus, they identified subacute and more chronic forms, with the latter generally lacking long-tract signs, and the more aggressive forms (survival $<3$ years) were presumed to be paraneoplastic though not consistently demonstrable.

PERM associated with only GlyR antibodies was reported in a 54-year-old man who presented with hyperekplexia, ${ }^{1}$ though no seizures or encephalopathy. His disease evolved over months, rather than the fulminant course described here, and prolonged treatment with a combination of high-dose IV methylprednisolone, plasma exchange, cyclophosphamide, and intrathecal baclofen for rigidity resulted in the patient eventually returning to work. GlyR antibodies have since been identified in patients with hyperekplexia, stiff-person syndrome, and PERM, usually without GAD antibodies. ${ }^{7,8}$

The additional features in our patient may relate to the coexistence of NMDAR antibodies. The core spectrum of NMDAR antibody-associated neuro- 
logic symptoms consists of an encephalitic picture, often in females, and includes seizures, movement disorders, autonomic instability, and reduction in consciousness. Initially, many cases were reported to have underlying ovarian teratoma. ${ }^{9}$ The study of a European cohort, however, suggested that the spectrum is somewhat wider with rigidity and ataxia, as seen in this patient, noted in 23\% and $9 \%$ of NMDAR-antibody positive patients, respectively. Only $20 \%$ of these patients had tumors, and overall $30 \%$ were men. ${ }^{2}$ An occult tumor, particularly testicular, was actively sought in our case but no evidence found macroscopically. However, we cannot definitively exclude this possibility.

The neuropathology of NMDAR encephalitis consists of mild meningeal and perivascular parenchymal lymphocytic inflammation, usually centered on the Sommer sector of the hippocampus and amygdala. However, involvement of brainstem and cerebellar structures, as seen in our case, has also been reported. ${ }^{9}$ We demonstrated direct apposition of CD8 + cytotoxic $\mathrm{T}$ cells to the cytoplasmic membrane of pyramidal and granule cell neurons of the hippocampus (figure 1) as well as, to a lesser degree, Purkinje cells. This observation is reminiscent of the picture seen in Rasmussen encephalitis. ${ }^{10,11}$ It is therefore possible that cytotoxic T-cell-mediated neuronal injury represents a common final pathway in a range of antibody-mediated encephalopathies. We also demonstrated the presence of scattered plasma cells in the meningeal and perivascular CNS parenchymal compartments, together with patchy immunoglobulin $\mathrm{G}$ deposition on neurons and glia and their processes. In contrast, parenchymal lymphocytes (excluding those in perivascular spaces) consisted of $\mathrm{T}$ cells, not $\mathrm{B}$ cells. This may suggest that autochthonic antibody production in meningeal or perivascular spaces may contribute to the ongoing immune reaction following an initial stimulus (possibly infectious) that has originated peripherally. Neuronal, glial, and diffuse neuropil immunoglobulin G deposition has been reported in association with diverse autoimmune encephalitides. ${ }^{9,12}$

A high density of NMDARs is found within the hippocampus. By contrast, GlyRs are mainly present in the brainstem and spinal cord. Thus we conclude that the hippocampal pathology reflects NMDAR antibody-mediated pathology, whereas the GlyR antibodies mediate the diencephalic histologic findings, although the second stage of NMDAR-antibody encephalitis also has features of a subcortical localization. $^{2}$ The coexistence of 2 antibodies, both of which are highly likely to be pathogenic, as they bind to extracellular domains of key neuronal receptors, raises the possibility that multiple autoan- tibodies exist in other patients. This case emphasizes the need for consideration of multiple antibody testing, and recognition of the possibility of sudden clinical deterioration. Further studies are needed to specifically address the potential role of aggressive immunotherapies.

\section{AUTHOR CONTRIBUTIONS}

Dr. Turner: drafting/revising the manuscript, study concept or design, analysis or interpretation of data, acquisition of data, study supervision. Dr. Irani: drafting/revising the manuscript, analysis or interpretation of data, contribution of vital reagents/tools/patients, acquisition of data. Dr. Leite: drafting/revising the manuscript, analysis or interpretation of data, acquisition of data. Dr. Nithi: drafting/revising the manuscript, study concept or design. Dr. Vincent: drafting/revising the manuscript, analysis or interpretation of data, contribution of vital reagents/tools/patients, study supervision. Dr. Ansorge: drafting/revising the manuscript, study concept or design, analysis or interpretation of data, contribution of vital reagents/tools/patients, acquisition of data, study supervision, obtaining funding.

\section{ACKNOWLEDGMENT}

The authors thank the parents of the patient for providing consent for postmortem study and publication and the staff at the Thomas Willis Oxford Brain Collection for their support.

\section{DISCLOSURE}

Dr. Turner receives royalties from the publication of The Brain: A Beginner's Guide (Oneworld, 2008) and Motor Neuron Disease: A Care Manual (Oxford University Press, 2010); serves as a consultant for Evalueserve, IMS Hospital Group Ltd., Smartanalyst Inc., Scisive, and Guidepoint Global; and receives research support from the Medical Research Council, the Motor Neurone Disease Association UK, and a Lady Edith Wolfson Clinician Scientist Fellowship. Dr. Irani has received a training grant from the National Institute of Health Research (NIHR), Department of Health, UK. Dr. Leite receives/has received research support from the Oxford NIHR Biomedical Research Centre, the National Commissioning Group, and the Sir Halley Stewart Trust, UK. Dr. Nithi reports no disclosures. Dr. Vincent has served on scientific advisory boards for the Patrick Berthoud Trust and the Myasthenia Gravis Foundation of America; has received funding for travel and a speaker honorarium from Baxter International Inc.; serves as an Associate Editor for Brain; receives royalties from the publication of Clinical Neuroimmunology (Blackwell Publishing, 2005); receives research support from the European Union, the Oxford NIHR Biomedical Research Centre, and Sir Halley Stewart Trust; and has received Musk antibody royalties and consulting fees from Athena Diagnostics, Inc., and Musk antibody royalties from RSR Ltd., Cardiff, UK. The University of Oxford, where A.V. is based, receives royalties and payments for antibody assays in neurologic diseases. Dr. Ansorge receives research support from the Oxford NIHR Biomedical Research Centre and UK Parkinson's Disease Society.

Received August 4, 2010. Accepted in final form January 19, 2011.

\section{REFERENCES}

1. Hutchinson M, Waters P, McHugh J, et al. Progressive encephalomyelitis, rigidity, and myoclonus: a novel glycine receptor antibody. Neurology 2008;71:1291-1292.

2. Irani SR, Bera K, Waters P, et al. N-methyl-D-aspartate antibody encephalitis: temporal progression of clinical and paraclinical observations in a predominantly nonparaneoplastic disorder of both sexes. Brain 2010;133: 1655-1667. 
3. Moersch FP, Woltman HW. Progressive fluctuating muscular rigidity and spasm ("stiff-man" syndrome); report of a case and some observations in 13 other cases. Proc Staff Meet Mayo Clin 1956;31:421-427.

4. Whiteley AM, Swash M, Urich H. Progressive encephalomyelitis with rigidity. Brain 1976;99:27-42.

5. Brown P, Marsden CD. The stiff man and stiff man plus syndromes. J Neurol 1999;246:648-652.

6. Goetz CG, Klawans HL. On the mechanism of sudden death in Moersch-Woltman syndrome. Neurology 1983; 33:930-932.

7. Mas N, Saiz A, Leite MI, et al. Anti-glycine-receptor encephalomyelitis with rigidity. J Neurol Neurosurg Psychiatry (in press 2011)

8. Clerinx K, Breban T, Schrooten M, et al. Progressive encephalomyelitis with rigidity and myoclonus
(PERM): resolution after thymectomy. Neurology 2011;76:303-304.

9. Dalmau J, Tuzun E, Wu HY, et al. Paraneoplastic anti-Nmethyl-D-aspartate receptor encephalitis associated with ovarian teratoma. Ann Neurol 2007;61:25-36.

10. Bien CG, Bauer J, Deckwerth TL, et al. Destruction of neurons by cytotoxic $T$ cells: a new pathogenic mechanism in Rasmussen's encephalitis. Ann Neurol 2002;51: 311-318.

11. Schwab N, Bien CG, Waschbisch A, et al. CD8+ T-cell clones dominate brain infiltrates in Rasmussen encephalitis and persist in the periphery. Brain 2009;132:1236-1246.

12. Tuzun E, Zhou L, Baehring JM, Bannykh S, Rosenfeld MR, Dalmau J. Evidence for antibody-mediated pathogenesis in anti-NMDAR encephalitis associated with ovarian teratoma. Acta Neuropathol Epub 2009.

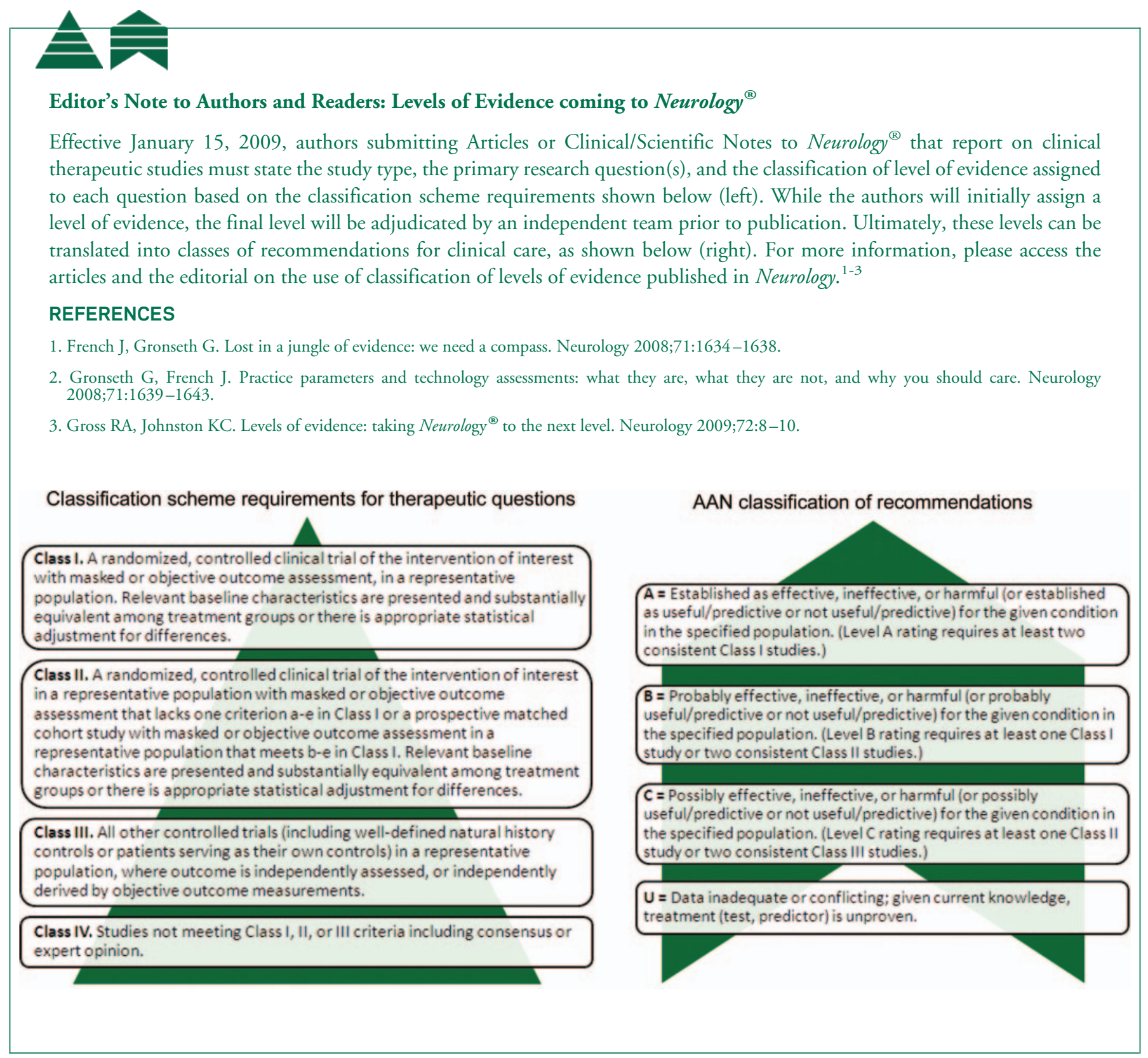

